\title{
Family Physician Perceptions of Their Role in Managing the Opioid Crisis
}

\author{
Laura Desveaux, $P T, P b D^{1,2}$ \\ Marianne Saragosa, RN, MN ${ }^{1}$ \\ Natasha Kitbulegoda, MPH ${ }^{1}$ \\ Noab Michael Ivers, $M D, P b D^{1}$ \\ 'Women's College Research Institute, \\ Toronto, Canada \\ ${ }^{2}$ Institute of Health Policy, Management \\ and Evaluation, University of Toronto, \\ Toronto, Canada \\ ${ }^{3}$ Department of Family and Community \\ Medicine, Women's College Hospital, \\ Toronto, Canada
}

\begin{abstract}
PURPOSE We examined the perspectives of family physicians (FPs) on opioid prescribing and management of chronic pain to better understand the barriers to safer prescribing in primary care and differences in perspectives that may be potential drivers of practice variation.
\end{abstract}

METHODS We used an exploratory qualitative study design. Semistructured interviews were conducted in June and July 2017 with 22 FPs in Ontario and coded inductively. Thematic analysis was used to identify themes, and a framework analysis explored the influence of physician demographics on prescribing experience.

RESULTS Three key themes emerged: the discrepancy between FPs' training and current practice, the tension between the FP's role and patient and system expectations, and the influence of length of time in practice and strength of therapeutic relationships on perspectives on opioid prescribing. There was an overarching sentiment among participants that FPs are unsupported in their efforts to manage chronic pain. More years in practice ( $\geq 15$ years) seems to influence practice patterns by increasing trust in therapeutic relationships and decreasing reliance on emergent guidelines (vs clinical experience).

CONCLUSION Number of years in practice influences FPs' response to emergent evidence, requiring initiatives to include strategies tailored to individual beliefs. Initiatives must move beyond dissemination and education to equip FPs with the skills they need to navigate emotionally charged conversations. External pressures and misaligned system and patient expectations place FPs at the center of a challenging situation, which may result in a higher risk of burnout compared with that of their specialist colleagues.

Ann Fam Med 2019;17:345-351. https://doi.org/10.1370/afm.2413.

\section{INTRODUCTION}

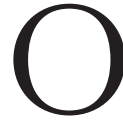
pioid prescription rates continue to rise ${ }^{1}$ despite increasing media attention on the opioid crisis. ${ }^{2,3}$ Family physicians (FPs) prescribe the greatest volume of opioids (22.9\%) and number of prescriptions $(31.2 \%)$ to patients with chronic noncancer pain (CNCP) ${ }_{1}^{4}$ making them an obvious target for quality improvement interventions. Primary care providers such as FPs are the main access point for the management of $\mathrm{CNCP}^{5,6}$ but medical training leaves many FPs feeling unprepared in this domain. ${ }^{78} \mathrm{New}$ guidelines and public health initiatives have emerged to target opioid prescribing in an attempt to support FPs in their management of $\mathrm{CNCP}^{9,10}$ yet the problem persists. Such initiatives are more likely to be successful if they address the context in which prescribing takes place and target the real-world circumstances of opioid prescribing in daily practice. ${ }^{11}$

Numerous qualitative studies have explored health care providers' experience of prescribing opioids, describing overarching themes of uncertainty, the need to balance the benefits and risks of opioid use, the primary aim of addressing pain, and fear of judgment, among others. ${ }^{8}$ These studies were conducted primarily in the United States, with only 3 such studies addressing opioid prescribing in Canada. Two were focused on long-term care and captured a multidisciplinary perspective with mini-
Laura Desveaux

Women's College Research Institute,

Women's College Hospital

76 Grenville Ave

Toronto, ON, Canada M5S 1B2

laura.desveaux@wchospital.ca 
mal physician input, ${ }^{12,13}$ and the other explored the perspectives of pain specialists. ${ }^{14}$ Prescribing behaviors vary by country, ${ }_{1}^{15}$ underscoring the need to better understand the perspectives of FPs in Ontario, Canada to inform local and national initiatives. Furthermore, previous qualitative work has been unable to identify drivers of practice variations ${ }^{16}$ to inform targeted support strategies to optimize impact within a resourceconstrained system.

To address this gap, we explored the perspectives of Canadian FPs on opioid prescribing and the management of CNCP. Our secondary objective was to explore differences in perspectives that may be potential drivers of practice variation. These insights will be used to inform system-wide initiatives aiming to support FPs and increase guideline adherence in primary care.

\section{METHODS}

\section{Study Design}

This qualitative study involved semistructured interviews of FPs in Ontario, Canada. The study was approved by the Women's College Hospital Research Ethics Board.

\section{Participants}

Family physicians were eligible if they were actively practicing and had experience in managing CNCP. A 2-part recruitment strategy was used to maximize the response rate. An initial recruitment e-mail was sent to FPs by Health Quality Ontario (HQO), a government agency that acts as the province's advisor on health care quality. ${ }^{17}$ An e-mail was sent to all FPs on HQO's distribution list who had requested to receive a practice report $(\mathrm{n}=924)$. A reminder e-mail was sent 2 weeks later. Interested FPs were asked to contact a member of the research team directly to ensure anonymity. Snowball sampling was also applied, whereby study participants were asked to identify colleagues who may have valuable insights, including those with divergent perspectives. Recruitment through personal networks of the study team was done to increase the sample pool and seek disconfirming cases. Participation was confidential, and all participants provided informed consent and received an honorarium for their participation.

\section{Data Collection}

Participants were offered the option of conducting the semistructured interview either in person (geography permitting) or by telephone, at their convenience. Interviews were conducted between July and September 2017 and continued until data saturation was reached and no new insights emerged. The interview guide included questions about participants' experiences of and challenges with prescribing opioid medications, individual beliefs about opioid prescribing, and sources of support for FPs. All interviews were recorded and transcribed verbatim. Transcripts were checked for accuracy against the audio file.

\section{Data Analysis}

Members of the research team included a practicing FP and health services researcher (N.M.I.), an implementation scientist with experience in qualitative methods who is clinically trained as a physical therapist (L.D.), a research coordinator with experience in qualitative methods who is clinically trained as a nurse (M.S.), and a research assistant with a master's degree in public health (N.K.). Interviews were conducted by a member of the team with no relationship to potential participants (M.S.) in order to mitigate bias.

Thematic analysis was used. We chose this technique because of its flexibility as an analysis method to identify themes in the data. ${ }^{18}$ Analysis started when 3 coders (M.S., N.K., and L.D.) independently read the transcripts repeatedly to achieve immersion and identify preliminary themes. The first several transcripts were coded by all 3 coders separately, and emergent codes were compared for consistency and to overcome the influence of individual bias. Once consistency was established, 2 team members (M.S. and N.K.) coded the remaining transcripts and met with a third team member (L.D.) at biweekly intervals to discuss the findings. Codes and associated narratives formed a codebook that was further analyzed and synthesized to develop the central themes. A meeting was then held with N.M.I. where he was invited to challenge the themes and pose alternative interpretations. Once the themes were fully developed, a framework analysis ${ }^{19,20}$ was used to explore whether certain physician demographics (ie, sex, years in practice, years in current practice, and geography) influenced the perceptions or experiences of participants. Concurrent data collection and data analysis processes allowed interview questions to be revised in order to further explore emerging insights. ${ }^{21}$

\section{RESULTS}

A total of 22 interviews were conducted with practicing FPs, of whom 12 were female. All interviews but 1 were conducted over the phone. Length of interviews ranged from 20 to 59 minutes (mean, 32 minutes). Physicians had a range of 2-32 years of practice experience (mean, 12 years). Twenty FPs worked in group practices, and 2 reported working alone (see Table 1 for participant demographics). 
Family physicians experience a tension adhering to guidelines while attempting to effectively manage patient symptoms, which creates a feeling of being caught in the middle of the opioid crisis. Confidence in prescribing, the burden of emotionally laden interactions, and guideline-concordant behavior were influenced by years in practice. There was an overarching sentiment among participants that a lack of supports to manage chronic pain or substance use disorder within the broader health system impedes guidelineconcordant care and effective management.

\section{Discrepancies Between FP Training and Current Expectations}

Family physicians emphasized that the prevailing wisdom before the opioid crisis was that chronic pain was undertreated, which encouraged a liberal approach to opioid prescribing. This approach was coupled with the notion that patients were the experts in their symptoms and pain management needs. The expectation was that opioid prescribing would be informed solely by the patient's subjective report. These circumstances have led to legacy patients who have been on opioid therapy for many years and are resistant to change.

Family physicians reflected on the recent evolution in external expectations and evidence related to opioid

Table 1. Participant Demographics

\begin{tabular}{lccccc}
\hline & & & & & Primary \\
ID & Sex & Age, Y & $\begin{array}{c}\text { Years in } \\
\text { Practice }\end{array}$ & Area & $\begin{array}{c}\text { Funding } \\
\text { Model }\end{array}$ \\
\hline P001 & F & 32 & 2 & Urban & Capitation \\
P002 & M & 37 & 5 & Urban & Capitation \\
P003 & M & 33 & 4 & Urban & Capitation \\
P004 & F & 46 & 17 & Rural & Capitation \\
P005 & F & 31 & 4 & Rural & Capitation \\
P006 & M & 32 & 4 & Urban & Capitation \\
P007 & M & 38 & 8 & Rural & Capitation \\
P008 & F & 37 & 9 & Urban & Capitation \\
P009 & F & 36 & 9 & Urban & Capitation \\
P010 & M & 31 & 2 & Urban & Capitation \\
P011 & F & 43 & 14 & Urban & Capitation \\
P012 & F & 38 & 9 & Urban & Capitation \\
P013 & F & 53 & 27 & Urban & Capitation \\
P014 & F & 54 & 27 & Rural & Capitation \\
P015 & M & 47 & 13 & Urban & Fee-for-service \\
P016 & F & 33 & 4 & Urban & Capitation \\
P017 & M & 61 & 32 & Urban & Capitation \\
P018 & M & 53 & 26 & Urban & Capitation \\
P019 & F & 31 & 4 & Urban & Capitation \\
P020 & M & 33 & 5 & Urban & Capitation \\
P021 & F & 38 & 10 & Urban & Fee-for-service \\
P022 & M & 58 & 25 & Rural & Fee-for-service \\
\hline F female; M male. & & & & \\
\hline
\end{tabular}

prescribing. The prevailing sentiment is that opioid prescribing is likely to increase the risk of addictive behavior and opioid misuse, which fostered hesitancy among many FPs to prescribe opioids.

\section{Tensions Between the FP's Role and Patient and System Expectations}

Family physicians described a conflict between their desired role as a healer and the emerging expectation to police opioid use and potential misuse. Family physicians explicitly described their primary motivations to both help their patients and do the right thing. Participants often felt uncomfortable managing the tension between offering symptom relief and avoiding potential harm. Practicing with suspicion and cynicism was complicating their ability to address patient needs. This tension led some FPs to question whether the management of CNCP (including prescribing opioids) should be their responsibility. Several FPs suggested centralizing the pain management process around a single provider (eg, a pain specialist) in an effort to provide comprehensive, consistent care across the system.

Participants stated that specialists or other prescribers often initiated opioid prescriptions, with subsequent management of these cases offloaded to the FP. Experiences involving specialists prescribing large quantities of opioids to patients with minimal communication with the FP were common. FPs felt "stuck" when they had minimal knowledge of the initial rationale for the prescription and these patients requested renewals.

\section{Effect of Length of Time in Practice and Strength of Therapeutic Relationships on Perspectives on Opioid Prescribing}

Physicians at the beginning of their career $(\leq 5$ years' experience) emphasized the need to establish therapeutic relationships and a systematic approach to clinical management that valued objective evidence. For these FPs, opioid-related conversations with patients were emotionally laden and threatened the integrity of the physician-patient relationship. Physicians reported feeling stressed by the challenge of managing tenuous relationships that could be exacerbated by incorporating recommended surveillance measures (ie, urine screening). They felt frustrated by an inability to effectively address patient needs without resorting to opioids because of a lack of appropriate pain management resources within the system. They felt uncomfortable in their perceived dual role of being a patient advocate and responding to emergent public health concerns.

Physicians with long-standing, stable practices $(\geq 15$ years) described strong therapeutic relationships and a clinical approach that prioritized effective management of patient symptoms. Confidence in clinical manage- 
ment was influenced primarily by past experiences and individual patient relationships. Knowing their patients reduced their concerns about aberrant behavior and the perceived need to implement enforcement measures. Similar to their younger colleagues, these physicians described a lack of available resources to support effective pain management in their practice.

A few physicians with varying degrees of experience described a strict approach to opioid prescribing that involved regimented use of opioid contracts and required objective evidence of pain as an indication to prescribe opioids. Given the challenges associated with tapering dosage, these physicians stated that they preferred to avoid opioid prescribing altogether, although many described unintended consequences of this approach (eg, diversion to illicit opioid use or to other prescribers). These self-described "militant" FPs described a major change in practice patterns toward complete avoidance of opioid prescribing.

Physicians with less experience often referenced the guidelines as informing their practice only in theory. They questioned the feasibility of implementing guidelines in resource-constrained family practice settings, feeling that they were "caught in the middle" between guidelines and patient care. Beyond justifying clinical decisions to patients, the utility of the guidelines was minimal in the absence of clear, actionable strategies and access to system resources. In contrast, external expectations and recommendations had minimal impact on approaches to clinical management among FPs with more practice experience. These physicians described the need for patient-facing materials and strategies that help engage specialists in order to comprehensively address the opioid crisis. Finally, those who implemented strict routines to avoid opioid-induced adverse events referenced the guidelines to support their approach.

\section{DISCUSSION}

Many FPs are struggling to balance system and patient expectations with recent guidelines, resulting in a significant emotional burden. Our findings show that the majority of FPs exhibit a general apprehension and reluctance to prescribe opioids, aligning with previous work ${ }^{7,22}$ and signaling a slight shift in overall perceptions compared with a recent qualitative synthesis by Toye et $\mathrm{al}^{8}{ }^{8}$ where the predominant concern was managing the patient's pain. Fears of opioid-related overdose, misuse, and diversion have prompted a pendulum swing with respect to pain management. ${ }^{22}$ Some participants described cases where this has led to the sudden discontinuation of opioids or termination of the physician-patient relationship, indicating that the reticence to prescribe opioids could exacerbate the crisis rather than address it.

Our findings build on previous qualitative work $^{8,16}$ by adding novel insights into variation in perspectives that may drive practice variation, specifically related to years in practice. In keeping with previous findings, ${ }^{23,24}$ FPs felt more confident prescribing opioids in the context of a trusting, long-standing patient-physician relationship. This attitude may reflect ingrained clinical habits, because the prevailing sentiment at the turn of the 21st century was that chronic pain is undertreated because physicians underuse major opioids. ${ }^{25}$ Unlearning clinical behaviors is challenging ${ }^{26}$; evidence often has little or no effect on deadoption of clinical behaviors. ${ }^{27}$ Interventions among FPs earlier in their career should explicitly target the skills and strategies necessary for opioid prescribing (ie, more nuanced explanations of how to navigate challenging conversations and reinforcement of their ability to provide guidelineconcordant care). In contrast, interventions tailored to FPs with more experience should focus on emerging evidence about opioid prescribing (ie, updating knowledge about specific clinical indications and dosages and highlighting the consequences of overprescribing or avoiding the use of opioids).

\section{Table 2. Supporting Qualitative Data for Primary Themes}

Theme 1: What FPs were taught vs current expectations

"I came out of school in [the 1990s]. At that point, we were undertreating chronic pain, so we were told. So we were quite gung-ho about not under-treating pain, and using opioids because they were supposedly safer than anti-inflammatories. And now, the pendulum has swung ... there's new evidence that it might actually not be doing them any good." P04

"I'm convinced we have to do things a lot safer, but if we go too far the other way, we're protecting our own licenses perhaps, and maybe controlling the street supply of opioids, but I don't know if it's the very best thing for patient care." P01

Theme 2: Navigating tensions between the FP's role and patient and system expectations

"I think there's [an expectation that] we need to be police officers at the same time as physicians, that's always a conflict. As a physician, I can't function if I don't believe my patients. But there's this overarching responsibility medically and legally for me to make sure I'm also suspicious of patients. So that's a very big internal struggle." P12

"It always goes back to the family doc. It's very rare [for anyone] to say, well, now I'll take this over, I'll prescribe their opioids [or manage their pain]. It's more often that they're like, here's some advice, go follow up with your family doctor." P04

"[Patients] probably [have an opioid started] sometime during their hospitalization and then they just continued on opioids because of pain related issues. Unfortunately they probably have escalating doses of opioid and nobody actually addressed that, or no one actually attempts to wean them down or try other interventions." P03

"I know that new recommendations have come out, I try but I'm not going to change my practice if I think that the patients are stable and well on them. . . I think if I'm accurate and they are truthful and they are stable, I don't think I'm going to make a huge effort to change it at this point in time." P017 
The emotional burden of opioid prescribing and challenging patient conversations are felt across physician specialties. ${ }^{22,28,29}$ Opioid prescribing represents a significant proportion of specialist prescribing, with $56 \%$ of newly initiated opioid treatments attributed to non-primary care specialties. ${ }^{30}$ Although prescriptions are often initiated elsewhere in the system, the prevailing sentiment is that the care of these patients is "dumped" on FPs. This practice may increase the emotional toll experienced by FPs, as they are caught in the middle of the system and are unable to resolve the perceived tension between patient-centeredness and evidence-based practice. ${ }^{31}$ Educational strategies to disseminate guideline recommendations may be inadequate unless they address the emotional aspects of these patient-provider interactions and facilitate

\section{Table 3. Supporting Qualitative Data for Variation in Perspectives That May Influence Physician Practice}

Physicians with $\leq 5$ years of practice experience

Challenges with clinical management; emotional component of patient interactions.

"There are obviously patients who probably leave me because they don't like my rules and go to another doctor. But when you see how upset these patients are and how unstable they are, it's hard to know how we should be doing, like instituting, all these new measures." P001 (2 years in practice)

"I think the challenge, for me, is when you talk about decreasing, or trying to, patients kind of look at you and say 'But I still have pain. What do I do?' And often, there are not many other options. I don't have anywhere else [to send them] ... . [so I] say yeah, I will do this for you. Sometimes you just don't have it. And I think, for me, that's the emotional part. . . You're caught between the college and trying to help this person, and the medical evidence and the lack of resources out there for people that should be there." P016 (4 years in practice)

"I find it's just challenging because I don't know what else to offer. It's more that you feel bad for these people because they are in pain and even though these medications aren't good for pain really, I don't know what else to do for them." P019 (4 years in practice)

Physicians with $\geq 15$ years of practice experience

Confident in the use of opioids in their practice; highlight the need for patient education.

"I feel like there should be some help for us in educating the public about keeping their use of opioids at the lowest possible level, it's your safety. That they shouldn't expect their pain to be zero because for chronic pain, it's probably not going to be possible to reach zero. If they can go from an 8 to a 5, that's already pretty amazing. I feel like there should be a bit more public awareness and education." P013 (27 years of practice)

"Because I don't have new patients, I have people I've known for 20, 25 years, I tend to have a lot of that background, to know, well, what's their addiction potential, what are their issues? Then, whether or not I think they're actually going to be more functional or less functional [on opioids], like, is this going to help you lay on the couch or is this going to help you go back to work?" P014 (27 years in practice)

"There isn't any patient support material. I just have the guidelines and I'm supposed to relay the information to them. And I'm relaying the information to a client that's very resistant to change. I have to be like a pharmaceutical rep. I have to detail the patient. I have to get them to buy into the risk of the high doses. I don't have any support material for that. I don't have any evidence or graphs or charts to present to the patient to say, 'Hey, if you're on a Benzo and a narcotic, you're at a higher risk of dying.'" P018 (26 years of practice)

Physicians who were self-described strict prescribers

"It isn't [a problem] any more. I got rid of those people. I stopped opioids on those people where it was a problem, or they left my practice and are probably getting it from another doctor. So, it's hard to know if it's successful. . . . I said, no, you broke the opioid contract I had you on and here's a tapering dose and that's it. And then sometimes I just don't see them again." P002 (5 years in practice)

"It's almost impossible to get them off [opioids], because you can't pry their pills from their fingers, from their cold, dead fingers. They just sort of latch onto them. And there are some people who try to minimize their dose, but there are other people who are constantly asking for more and more and more, because their pain is not controlled. And it's not that they're not getting enough, it's that their pain is never going to be controlled by opioids." P015 (13 years in practice) development of the skills needed to navigate the resulting tensions. In the absence of tailored supports, guidelines may exacerbate variations in care and have unintended effects on FPs' self-efficacy. At a time when physicians are already experiencing unprecedented rates of work-related stress and burnout, feelings of being "caught in the middle" without adequate system supports may compromise the objectives of the Quadruple Aim (specifically clinician and patient experience). Physicians are under unprecedented pressure, and work-related stress and increased anxiety can erode physician resilience, ${ }^{32}$ placing providers at risk of burnout. Time pressures, lack of resources, and challenging patients are further threats to resilience, ${ }^{32}$ underscoring the need for interventions to include avigating challenging conversations as part of a multifaceted strategy.

Family physicians continue to feel unsupported in their management, ${ }^{33,34}$ which interferes with the ability to provide guidelineconcordant care. In addition to tailored educational strategies for primary care, supports to facilitate shared decision making, including additional specialist and allied health capacity, are necessary to address the present crisis. ${ }^{35}$ Furthermore, participants echoed the need for patientfacing strategies ${ }^{36}$ to address existing tensions and knowledge discrepancies. To address the opioid crisis at a population level, initiatives must adopt a comprehensive, multilevel strategy that targets all potential prescribers and the general public.

Some limitations of this work warrant highlighting. We acknowledge that conducting interviews via telephone limits the ability to interpret contextual and nonverbal data. Although data saturation was reached in our data sample ${ }^{37}$ and contrasting perspectives were highlighted, the results are not intended to reflect the general FP population in Ontario, Canada or beyond. It is important to note that this study was designed to solicit in-depth views from participants, and future work should explore whether these findings are consistent across the broader population of FPs throughout Canada. Several of our findings align with previous qualita- 
tive work, ${ }^{8,16,36}$ however, suggesting that a broader physician audience may endorse these findings. Some differences were noted in the experiences of rural vs urban $\mathrm{FPs}_{i}$ however, we were unable to rigorously investigate these differences given the small number of rural FPs we were able to recruit. The impact of practice setting (urban vs rural) and available clinic resources warrants further exploration. Finally, data were analyzed inductively in the absence of an a priori framework. Other analytical approaches may have rendered different interpretations.

Family physicians are a central access point for pain management and therefore a key target for strategies to implement safer, guideline-concordant prescribing practices. ${ }^{38-40}$ Our findings highlight how the number of years in practice influences how FPs respond to emergent evidence. External pressures and misaligned system and patient expectations place FPs at the center of a challenging situation, which may increase their risk of burnout compared with their specialist colleagues. Initiatives aiming to support safe opioid prescribing and guideline-concordant care among FPs must tailor content to the $\mathrm{FPs}^{\prime}$ prescribing practices, addressing their beliefs about the clinical utility of opioids and equipping them with the skills they need to navigate emotionally charged conversations with patients. To comprehensively address the opioid crisis at a system level, interventions are needed to facilitate shared decision making, improve specialist communication with primary care, and target high prescribers across specialties.

To read or post commentaries in response to this article, see it online at http://www.AnnFamMed.org/content/17/4/345.

Key words: opioids; pain management; family medicine

Submitted July 19, 2018; submitted, revised, February 1, 2019; accepted March 3, 2019.

Funding support: N.M.I. is supported by a New Investigator Award from the Canadian Institutes of Health Research (CIHR) and a Clinician Scientist Award from the Department of Family and Community Medicine at the University of Toronto. This work was supported by CIHR and the Government of Ontario via a grant from the Ontario Strategy for Patient-Oriented Research Support Unit.

Acknowledgments: The authors acknowledge Tara Kiran for her thoughtful feedback on the manuscript and the participating physicians and Health Quality Ontario for their support and involvement.

\section{References}

1. Canadian Institute for Health Information. Pan-Canadian Trends in the Prescribing of Opioids, 2012 to 2016. Ottawa, ON: Canadian Institute for Health Information; 2017.

2. Fischer B, Russell C, Murphy Y, Kurdyak P. Prescription opioids, abuse and public health in Canada: is fentanyl the new centre of the opioid crisis? Pharmacoepidemiol Drug Saf. 2015;24(12):1334-1336.
3. Government of Canada. Opioid crisis in Canada. https://www canada.ca/en/services/health/campaign/drug-prevention.html1. Published 2018. Accessed Feb 15, 2018.

4. Ringwalt C, Gugelmann H, Garrettson M, et al. Differential prescribing of opioid analgesics accordin $\mathrm{g}$ to physician specialty for Medicaid patients with chronic noncancer pain diagnoses. Pain Res Manag. 2014;19(4):179-185.

5. Marcus DA. Practical approach to the management of chronic pain. Compr Ther. 2005;31(1):40-49.

6. Reid MC, Engles-Horton LL, Weber MB, Kerns RD, Rogers EL, $\mathrm{O}^{\prime}$ Connor PG. Use of opioid medications for chronic noncancer pain syndromes in primary care. J Gen Intern Med. 2002;17(3):173-179.

7. Upshur CC, Luckmann RS, Savageau JA. Primary care provider concerns about management of chronic pain in community clinic populations. J Gen Intern Med. 2006;21(6):652-655.

8. Toye F, Seers K, Tierney S, Barker KL. A qualitative evidence synthesis to explore healthcare professionals' experience of prescribing opioids to adults with chronic non-malignant pain. BMC Fam Pract. 2017;18(1):94.

9. Busse JW, Craigie S, Juurlink DN, et al. Guideline for opioid therapy and chronic noncancer pain. CMAJ. 2017;189(18):E659-E666.

10. Ontario Drug Policy Research Network. Behind the prescriptions: a snapshot of opioid use across all Ontarians. http://odprn. ca/wp-content/uploads/2017/08/ODPRN-Report_Behind-thePrescriptions.pdf. Published Aug 22, 2017. Accessed Feb 15, 2018.

11. Baker R, Camosso-Stefinovic J, Gillies C, et al. Tailored interventions to overcome identified barriers to change: effects on professional practice and health care outcomes. Cochrane Database Syst Rev. 2010;3(3):CD005470.

12. Kaasalainen S, Brazil K, Coker E, et al. An action-based approach to improving pain management in long-term care. Can J Aging. 2010; 29(4):503-517.

13. Kaasalainen S, Coker E, Dolovich L, et al. Pain management decision making among long-term care physicians and nurses. West J Nurs Res. 2007;29(5):561-580; discussion 581-568.

14. Chang Y, Zhu KL, Florez ID, et al. Attitudes toward the Canadian Guideline for Safe and Effective Use of Opioids for Chronic NonCancer Pain: a qualitative study. J Opioid Manag. 2016;12(6):377-387.

15. Tsiantou V, Moschandreas J, Bertsias A, et al. General practitioners' intention to prescribe and prescribing patterns in selected European settings: the OTCSOCIOMED project. Health Policy. 2015;119(9): 1265-1274.

16. Seamark D, Seamark C, Greaves C, Blake S. GPs prescribing of strong opioid drugs for patients with chronic non-cancer pain: a qualitative study. Br J Gen Pract. 2013;63(617):e821-828.

17. Health Quality Ontario. http://www.hqontario.ca/.

18. Vaismoradi M, Turunen H, Bondas T. Content analysis and thematic analysis: implications for conducting a qualitative descriptive study. Nurs Health Sci. 2013;15(3):398-405.

19. Gale NK, Heath G, Cameron E, Rashid S, Redwood S. Using the framework method for the analysis of qualitative data in multidisciplinary health research. BMC Med Res Methodol. 2013;13(1):117.

20. Smith J, Firth J. Qualitative data analysis: the framework approach. Nurse Res. 2011;18(2):52-62.

21. Thorne S. Data analysis in qualitative research. Evid Based Nurs. 2000;3(3):68-70.

22. Hurstak EE, Kushel M, Chang J, et al. The risks of opioid treatment: perspectives of primary care practitioners and patients from safetynet clinics. Subst Abus. 2017;38(2):213-221.

23. Bergman AA, Matthias MS, Coffing JM, Krebs EE. Contrasting tensions between patients and PCPs in chronic pain management: a qualitative study. Pain Med. 2013;14(11):1689-1697. 
24. Matthias MS, Parpart AL, Nyland KA, et al. The patient-provider relationship in chronic pain care: providers' perspectives. Pain Med. 2010;11(11):1688-1697.

25. Moulin DE, Clark AJ, Speechley M, Morley-Forster PK. Chronic pain in Canada-prevalence, treatment, impact and the role of opioid analgesia. Pain Res Manag. 2002;7(4):179-184.

26. Carroll AE. It's hard for doctors to unlearn things; that's costly for all of us. New York Times. September 11, 2018:B5.

27. Niven DJ, Rubenfeld GD, Kramer AA, Stelfox HT. Effect of published scientific evidence on glycemic control in adult intensive care units. JAMA Intern Med. 2015;175(5):801-809.

28. Calcaterra SL, Drabkin AD, Leslie SE, et al. The hospitalist perspective on opioid prescribing: a qualitative analysis. J Hosp Med. 2016; 11(8):536-542.

29. Kim HS, McCarthy DM, Hoppe JA, Mark Courtney D, Lambert BL. Emergency department provider perspectives on benzodiazepineopioid coprescribing: a qualitative study. Acad Emerg Med. 2018; 25(1):15-24.

30. Health Quality Ontario. Starting on opioids: opioid prescribing patterns in Ontario by family doctors, surgeons, and dentists, for people starting to take opioids. http://startingonopioids.hqontario.ca

31. Hoffmann TC, Montori VM, Del Mar C. The connection between evidence-based medicine and shared decision making. JAMA. 2014; 312(13):1295-1296.

32. Cheshire A, Ridge D, Hughes J, et al. Influences on GP coping and resilience: a qualitative study in primary care. Br J Gen Pract. 2017; 67(659):e428-e436.
33. Clark LG, Upshur CC. Family medicine physicians' views of how to improve chronic pain management. J Am Board Fam Med. 2007; 20(5):479-482.

34. Barry DT, Irwin KS, Jones ES, et al. Opioids, chronic pain, and addiction in primary care. J Pain. 2010;11(12):1442-1450.

35. Légaré $F$, Ratté $S$, Stacey $D$, et al. Interventions for improving the adoption of shared decision making by healthcare professionals. Cochrane Database Syst Rev. 2010;5(5).

36. McCrorie C, Closs SJ, House A, et al. Understanding long-term opioid prescribing for non-cancer pain in primary care: a qualitative study. BMC Fam Pract. 2015;16:121

37. Etikan I, Musa SA, Alkassim RS. Comparison of convenience sampling and purposive sampling. American Journal of Theoretical and Applied Statistics. 2016;5(1):1-4.

38. College of Physicians and Surgeons of Ontario. Avoiding abuse, achieving a balance: tackling the opioid public health crisis. Toronto, ON: College of Physicians and Surgeons of Ontario; 2010.

39. Kotalik J. Controlling pain and reducing misuse of opioids: ethical considerations. Can Fam Physician. 2012;58(4):381-385, e190-e195.

40. Morley-Forster PK, Clark AJ, Speechley M, Moulin DE. Attitudes toward opioid use for chronic pain: a Canadian physician survey. Pain Res Manag. 2003;8(4):189-194. 